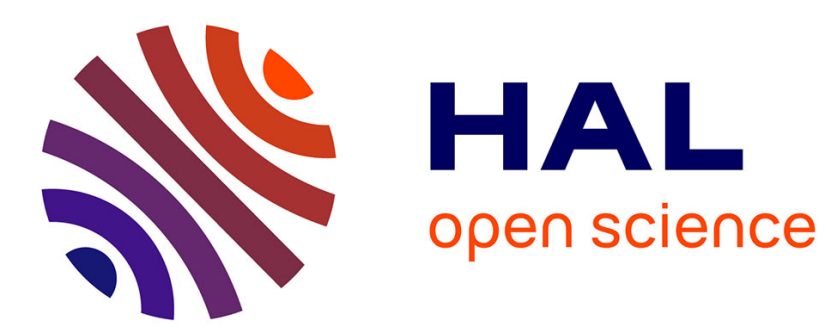

\title{
Individuation référentielle de la construction infinitive V-TESSA en finnois: entre détermination nominale et complémentation verbale
}

Outi Duvallon

\section{- To cite this version:}

Outi Duvallon. Individuation référentielle de la construction infinitive V-TESSA en finnois: entre détermination nominale et complémentation verbale. Antoine Gautier; Eva Havu; Dan Van Raemdonck. DéterminationS, P.I.E Peter Lang, 2016, 978-2-87574-326-8. halshs-01341838

\section{HAL Id: halshs-01341838 \\ https://shs.hal.science/halshs-01341838}

Submitted on 5 Jul 2016

HAL is a multi-disciplinary open access archive for the deposit and dissemination of scientific research documents, whether they are published or not. The documents may come from teaching and research institutions in France or abroad, or from public or private research centers.
L'archive ouverte pluridisciplinaire HAL, est destinée au dépôt et à la diffusion de documents scientifiques de niveau recherche, publiés ou non, émanant des établissements d'enseignement et de recherche français ou étrangers, des laboratoires publics ou privés. 
DUVALLON, Outi, 2016, «Individuation référentielle de la construction infinitive V-TESSA en finnois : entre détermination nominale et complémentation verbale » in Gautier A., Havu E., \& Van Raemdonck D. (éds), DéterminationS, Bruxelles, Peter Lang, pp. 163-180.

Outi Duvallon

INALCO/USPC, CNRS UMR8202 IRD UMR135, SeDyL

Courriel : outi.duvallon@inalco.fr

\section{Individuation référentielle de la construction infinitive $V$-TESSA en finnois : entre détermination nominale et complémentation verbale ${ }^{1}$}

\section{Introduction}

Cet article abordera la question de la détermination en examinant une construction finnoise qui peut être considérée comme un converbe (Herlin et Kotilainen, 2005, p. 259) : il s'agit d'une forme verbale non finie qui permet de construire une subordination de type adverbial (Haspelmath, 1995 ; Creissels, 2006, p. 227). Un premier exemple en est donné en (1a) où l'infinitif (mis en gras) exprime un procès qui sert de repère au procès exprimé par le verbe principal. Le rapport entre les deux procès est celui de coïncidence temporelle :

1a)

Katso-e-ssa-ni $\boldsymbol{i}_{\mathrm{i}} \quad$ si-tä tarke-mm-in huomas-i- $n_{\mathrm{i},} \quad$ että (...) regarder-INF-INE-POS.1 la-PAR attentif-COMP-INS s'apercevoir-PRÉT-1 CONJ

« En la regardant de plus près, je me suis aperçu que (...)»

1b)

Kun katso-i-n $n_{\mathrm{i}}$ si-tä tarke-mm-in, huomas-i- $n_{\mathrm{i}}, \quad$ että (...) Quand regarder-PRÉT-1 la-PAR attentif-COMP-INS s'apercevoir-PRÉT-1 CONJ

«Quand je l'ai regardée de plus près, je me suis aperçu que (...)»

Les grammaires du finnois nomment cette construction infinitive qui est identifiable par la marque TESSA construction temporelle ${ }^{2}$ (fi. temporaalirakenne) et la présentent en règle générale comme un équivalent synthétique d'une subordonnée temporelle telle qu'illustrée en (1b) (Setälä, 1891 ; Vilkuna, 1996, p. 309 ; ISK, 2004, § 543) 3 .

En finnois standard, la construction temporelle en TESSA se caractérise par un degré de phrasticité élevé (Hakulinen et Karlsson, 1979, p. 337-338 ; Fernandez-Vest, 2008). En (1a), l'infinitif est accompagné d'un complément d'objet, sitä «la », et d'un modifieur adverbial, tarkemmin « de plus près », comme le verbe fini en (1b). Quant à l'argument sujet de la base verbale, il est exprimé dans cet exemple par un suffixe possessif, -ni, coréférent avec le sujet du verbe principal.

D'un autre côté, si on examine des données représentant des variétés non standard, on trouve des occurrences de l'infinitif en TESSA qui relèvent d'un emploi plutôt substantif.

\footnotetext{
${ }^{1}$ Nous tenons à remercier les relecteurs anonymes pour leurs remarques pertinentes.

${ }^{2}$ La marque TESSA a plusieurs allomorphes : essa, essä, issa, issä, dessa, dessä, tessa, tessä, lessa, lessä, ressa, ressä, nessa, nessä. Notons aussi qu'il existe une forme en TUA, que nous n'étudierons pas ici, qui indique un procès antérieur au procès exprimé par le verbe principal (voir ISK 2004, § 543).

${ }^{3}$ La tradition grammaticale finnoise utilise le terme lauseenvastike, littéralement « substitut de phrase », qui a été traduit en français par quasi-proposition (Sauvageot 1949, p. 139 ; 1973, p. 93, 392 ; voir aussi FernandezVest 2008).
} 
L'infinitif peut être précédé d'un démonstratif atone, siinä en (2), dont la fonction s'apparente à celle d'un déterminant nominal :

2)

miten lie siinä lähti-e-ssä haihtu-nut miele-stä...

comment AUX-POT-3 DÉM.INE partir-INF-INE s'évaporer-PPA esprit-ÉLA

« je ne sais pas comment ça m'est sorti de la tête au moment de partir...»

La problématique qui sera étudiée ici est celle de savoir comment se construit la valeur référentielle de l'infinitif. On s'interrogera sur les procédés par lesquels, à partir d'une base verbale lexicalisant un type de procès, est établie, moyennant les marques qui s'y ajoutent et les compléments qui l'accompagnent, une valeur référentielle spécifique, l'idée d'un procès impliquant des participants et ayant des coordonnées spatio-temporelles. La notion de détermination aura un rôle double : d'une part, il s'agira de la mise en relation de deux procès, celui exprimé par l'infinitif ayant une fonction cadrative - donc de détermination - par rapport à la prédication principale, et d'autre part, il s'agira de déterminations du procès cadratif luimême. Une attention particulière sera portée à l'unité formée par le démonstratif siinä et l'infinitif en TESSA, qui n'a pas suscité l'intérêt des grammairiens. Notre objectif est de définir la spécificité discursive du procédé de détermination nominale par rapport à la construction de type phrastique aussi bien du point de vue de la syntaxe interne que de celui de la syntaxe externe de la construction infinitive.

Dans ce qui suit seront d'abord présentés les suffixes qui composent la marque TESSA dont est porteuse la forme verbale non finie. Nous passerons ensuite à la question de la construction de la valeur référentielle de l'infinitif selon deux types de procédés, nominal et verbal, et enfin, la dernière partie de l'article sera consacrée au rôle déterminatif du démonstratif siinä.

\section{Les composants de la marque TESSA}

La marque TESSA est formée de la concaténation de deux suffixes. Le premier élément, $T E$, est un suffixe de nominalisation qui s'ajoute à une base verbale pour permettre ensuite l'adjonction d'un suffixe casuel, en l'occurrence, le suffixe inessif ssA.

3)

base verbale $(\mathrm{V})+$ suffixe de nominalisation $T E+$ suffixe inessif $s s A$

\subsection{Le suffixe inessif $s s A$}

Le suffixe inessif fonctionne comme relateur qui établit une relation entre deux termes, $\mathrm{X}$ et Y (cf. Duvallon et de Penanros, 2015). Y, terme repère, est lexicalisé par la base du suffixe inessif tandis que $\mathrm{X}$, terme repéré, est un élément externe du syntagme à l'inessif. Dans le cas de la construction temporelle, $\mathrm{X}$ correspond à l'évènement exprimé par la proposition principale (4) :

4)

$\begin{array}{cccc}\text { [odotta-e-ssa-an ] } & {[\text { hän }} & \text { hymyile-e }] \mathbf{x} \\ \text { attendre-INF-INE-POS. } 3^{4} & \text { il } & \text { sourire-3 } \\ \text { « [en attendant] } \mathbf{Y}[\text { il sourit }] \mathbf{X} » & \end{array}$

\footnotetext{
${ }^{4}$ Pour le suffixe possessif, voir la partie 3.1.
} 
$\mathrm{X}$ est repéré par Y, c'est-à-dire que le terme Y est une source de déterminations pour le terme $\mathrm{X}$.

Pour donner une définition de la sémantique du cas inessif, nous nous inspirons de l'analyse de la préposition dans en français proposée par Franckel et Paillard (2007, p. 152) : dans le repérage opéré par le cas inessif, le terme repère $\mathrm{Y}$ peut être considéré comme «ce qui tient lieu d'univers » du terme repéré $X$. La relation ainsi établie est de nature abstraite, ce qui veut dire que la valeur précise des groupes nominaux à l'inessif dépend du contenu lexical de la base du suffixe $s s A$, mais aussi de la nature du terme X. Le terme repère Y peut correspondre à un univers spatial, mais aussi à un univers temporel, situationnel, qualificatif, ou autre. Étant donné que dans la construction temporelle, la base du suffixe inessif est d'origine verbale, il s'agit d'un univers évènementiel, et par là-même d'un univers temporel, un évènement étant censé occuper un certain espace temporel (cf. Larjavaara, 1990, p. 192).

Il faut encore ajouter une caractéristique du suffixe inessif. En plus de fonctionner comme relateur, il contribue à construire une occurrence de la notion lexicalisée par sa base, dotée d'un ancrage spatio-temporel (cf. Culioli, 1999, p. 10-11, 14-15 ; Kleiber, 2011). Ce qui importe ici, c'est que le terme $\mathrm{Y}$, en l'occurrence l'évènement repère, a une autonomie première par rapport au terme $\mathrm{X}$, l'évènement repéré.

\subsection{La marque de l'infinitif $T E$}

L'élément $T E$ qui se trouve entre la base verbale et le suffixe inessif est considéré par les grammaires comme une marque de l'infinitif. En effet, une base verbale en $T E$ se situe entre la catégorie des noms et celle des verbes. À la différence des noms déverbatifs, elle n'accepte qu'un nombre limité de suffixes casuels et n'a pas la forme nominative. Cependant, on peut supposer qu'elle a connu, dans un état antérieur de la langue, un emploi plus productif en tant que nom (cf. Saukkonen, 1965, p. 22-24). Il s'agirait ainsi d'une évolution connue dans différentes langues dans laquelle les infinitifs se développent à partir de noms déverbatifs (Jespersen, 1924, p. 139-140 ; Ravila, 1945, p. 157 ; Cresseils, 2006, p. 231).

L'origine nominale de l'infinitif en TESSA s'observe dans certains de ses emplois où il entre dans une relation de paraphrase, ou reformulation, avec un véritable nom d'action (cf. Saukkonen, 1965, p. 27 ; Kiuru, 1981, p. 2-3 ; Herlin et Kotilainen, 2005, note 9). Dans l'exemple (5a), qui est un proverbe illustrant un emploi relativement ancien de la forme en TESSA, cette dernière peut être reformulée à l'aide d'un nom en minen, suffixe déverbatif productif en finnois moderne, qui prend la forme de mise- devant le suffixe inessif (5b). Dans les exemples (6a) et (6b), relevés de textes contemporains, l'importance pour un politicien d'être sûr de soi est prise en considération du point de vue d'une situation particulière, à savoir les apparitions publiques, exprimée par un complément circonstanciel au cas inessif. En (6a) la base du suffixe ssä est l'infinitif en $T E$, alors qu'en (6b), il s'agit d'un nom déverbatif en minen $^{5}$ :

5a)

Aika mene-e arvel-le-ssa.

temps passer-3 tergiverser-INF-INE

« Le temps passe en tergiversant. »

\footnotetext{
${ }^{5}$ Nous entendons par paraphrase une relation de parenté formelle entre deux expressions. Cette équivalence linguistique ne signifie pas une identité totale, mais une « identité d'un certain point de vue » (cf. Fuchs, 1982, p. 28). Ainsi, si les deux formes nominales en (6a) et en (6b) construisent comme référent un type de procès, l'infinitif en $T E$ met plus nettement l'accent sur l'idée du procès en accomplissement (« lorsqu'il apparaît en public ») que ne le fait le nom déverbatif en minen qui peut s'interpréter aussi comme désignant le résultat de ce procès («dans ses apparitions publiques, dans sa façon d'apparaître en public »).
} 
$5 b)$

'Aika mene-e arvele-mise-ssa.'

temps passer-3 tergiverser-NMLS-INE

«'Le temps passe en tergiversation.' »

6a)

Tärkeintä on, että parlamentaarikko (...) on itsevarma esiinty-e-ssä-än.

parlementaire être-3 sûr de lui apparaître en public-INF-INE-POS.3

« Le plus important est que le parlementaire soit sûr de lui lorsqu'il apparaît en public. »

(http://m.kaleva.fi/taytta-laukkaa/..., 2004)

6b)

Poliitiko-n pitä- $\ddot{a}$ ol-la(...) itsevarma esiinty-mise-ssä-än .

politicien-GEN devoir-3 être-INF sûr de lui apparaître en public-NMLS-INE-POS.3.

« Le politicien doit être sûr de lui dans ses apparitions publiques.

(http:/blogit iltalehti.fi/..., 2012)

\section{La valeur référentielle}

L'origine nominale de l'infinitif en TESSA conditionne aussi la façon dont s'établit sa valeur référentielle. La base verbale lexicalise une notion, comme celle de « regarder » en (1a) ci-dessus. Grâce au suffixe de nominalisation, le procès est évoqué de façon condensée, comme une « chose », au lieu d'être décrit comme un scénario qui se déploie progressivement avec sa temporalité propre et qui est ancré dans un système temporel et modal construit en relation avec la situation d'énonciation (cf. De Vogüé, 2005 ; Buridant, 2005 ; Honeste, 2007). L'infinitif en TESSA, bien qu'il implique la notion de temporalité, ne comporte pas d'informations sur des coordonnées temporelles et modales du procès ; l'interprétation de celles-ci dépend des indications contextuelles.

\subsection{Forme de reprise sur le modèle dialectal}

Dans les dialectes finnois, l'infinitif en TESSA connaît un emploi plus restreint que celui dont elle fait l'objet dans le finnois écrit standard. Cette forme s'utilise pour évoquer un évènement qui a déjà été mentionné, explicitement ou implicitement, dans le discours ou qui fait partie des connaissances générales du monde, comme par exemple le lever du jour, le coucher de soleil, la prise de repas, etc. (Lindén, 1961, p. 194 ; Herlin et Kotilainen, 2005, p. 265-266).

Le cas de figure typique d'un texte narratif est celui où l'infinitif fonctionne comme forme de reprise d'un prédicat verbal (cf. Buridant, 2005, p. 113). Dans l'exemple (7), extrait d'une œuvre littéraire, le verbe repris est odottaa «attendre »:

7)

1 Rovasti seisoo kammarinsa ikkunassa (...).

«Le pasteur se tient à la fenêtre de sa chambre (...). »

2 Hän on katsel-lut ja odotta-nut, tokko harakka uskaltaisi siepata jotakin.

il AUX-3 regarder-PPA et attendre-PPA

« Ça fait un moment qu'il regarde et attend [de voir] si la pie osera attraper quelque chose. »

3 Ja odotta-e-ssa-an hän $_{\mathrm{i}}$ hymyile-e...

et attendre-INF-INE-POS.3 il sourire-3

« Et en attendant, il sourit... »

(J. Aho, 1884 [2011], Rautatie, p. 19) 
L'infinitif sert donc à repérer la prédication principale par un évènement connu qui ne fait pas partie de l'apport de l'énoncé. Dans ce type d'emploi, la construction formée autour de l'infinitif est peu complexe. L'infinitif peut être utilisé seul, sans aucune indication sur les participants du procès :

8)

1 Säiden suhteen reissu oli varsin uskomaton.

«Du point de vue de la météo, le voyage était assez incroyable. »

2 Men-ne-ssä ja tul-le-ssa sato-i, mutta muuten koko kahden viikon aikana aller-INF-INE et revenir-INF-INE pleuvoir-PRÉT-3

" Il a plu à l'aller et au retour, mais sinon pendant les deux semaines, »

3 ei satanut kuin kerran ja lämpötila pysyi hellelukemissa lähes joka päivä.

« il n'a plu qu'une seule fois et les températures étaient aux alentours des 25 degrés presque tous les jours. »

(http://www.kohinaa.com/..., 2006)

En (8), les formes mennessä «à l'aller » et tullessa « au retour» ne portent pas de marque spécifiant l'argument sujet de la base verbale, mais l'interprétation de celui-ci n'est pas pour autant arbitraire, non spécifique : il est question d'un aller et d'un retour définis impliquant les participants du voyage mentionné dans le contexte (ligne 1). Cet exemple peut être comparé au proverbe en (5a) ci-dessus où l'infinitif en TESSA, qui désigne de manière générique un type de procès, implique un argument sujet non spécifique («Le temps passe quand on - n'importe qui - tergiverse. »).

L'une des particularités de l'infinitif en TESSA est en effet que la base verbale est toujours un verbe personnel qui met en jeu un argument sujet, support du procès. Lorsque l'argument sujet est spécifique et, de plus, identique au sujet du verbe principal de l'énoncé, il peut - mais ne doit pas - avoir comme réalisation un suffixe possessif, comme en (7) ci-dessus où l'infinitif odotta-e-ssa-an «en attendant» est porteur du suffixe possessif de troisième personne -an coréférent avec le pronom sujet hän «il»; en (9), il s'agit du suffixe possessif de première personne du singulier $-n i^{6}$ :

9)

Tien huonouden korvasi (...) meitä ympäröivä luonto.

«Le mauvais état de la route était compensé (...) par la nature qui nous entourait. »

$E-n_{\mathrm{i}}$ ole sellais-ta maisema-a vielä elä-i-ssä-ni $\boldsymbol{i}_{\mathrm{i}}$ näh-nyt.

NEG-1 AUX tel-PAR paysage-PAR encore vivre-INF-INE-POS.1 voir-PPA

« $\mathrm{Je}_{\mathrm{i}} \mathrm{n}$ 'avais pas encore vu un tel paysage dans mai vie. »

(J. Aho, 1911, Sanomalehtimiesajoiltani)

L'individuation explicite de la référence se fait donc par l'identification des participants du procès. La récupération d'un argument sujet qui a une réalisation lexicale et qui n'est pas identique au sujet du verbe principal s'appuie sur le procédé de nominalisation qui consiste à transposer le cas nominatif en génitif (cf. Benveniste, 1966, p. 147). Ainsi, au sujet lexical du verbe fini qui est au nominatif, tel que päivä «jour » en (10a), correspond un complément au génitif qui est antéposé à l'infinitif, päivä- $n$ en (10b); on observe la même chose avec un nom déverbatif en minen en (10c) :

\footnotetext{
${ }^{6}$ Comme illustration de l'absence de suffixe possessif dans le cas de la coréférence entre l'argument sujet de l'infinitif et le sujet du verbe principal, voir ci-dessous les ex. 11a et 12a.
} 
10a)

päivä valkene-e

jour.NOM blanchir-3

« le jour se lève »

$10 \mathrm{~b})$

Päivä-n vale-te-ssa alko-i Matti kuulustel-la Liisa-a koti-in tule-va-ksi. jour-GÉN blanchir-INF-INE commencer-PRÉT-3 Matti attendre-INF Liisa-PAR maison-INE venir-PPR-TRA «Au lever du jour, Matti commença à attendre le retour de Liisa à la maison. » (J. Aho, 1884 [2011], Rautatie, p. 69)

10c)

päivä-n valkene-minen

jour-GÉN blanchir-NMLS.NOM

« [le] lever du jour»

La construction de l'infinitif peut comporter aussi un élément qui participe à la description lexicale de l'évènement et qui correspond à un complément nucléaire de la base verbale, tel que kouluun «à l'école », complément de lieu qui spécifie le verbe de mouvement mennä «aller» en (11a), ou puolista «repas de midi», complément d'objet spécifiant syödä «manger » en (12a) (Lindén, 1961, p. 197-198; Kiuru, 1981, p. 2) :

11a)

Seuraava-na päivä-nä hän astu-i hyvin hitaasti koulu-un men-ne-ssä (...) suivant-ESS jour-ESS elle marcher-PRÉT-3 très lentement école-ILL aller-INF-INE «Le lendemain, elle marchait très lentement en allant à l'école $(. .)$.

(M. Canth, 1886, Hanna)

11b)

hän men-i koulu-un

elle aller-PRÉT-3 école-ILL

« elle allait à l'école »

12a)

Puolis-ta syö-de-ssä ol-i Liisa hyvin huol-i-ssa-an ole-va-n näköinen. repas de midi-PAR manger-INF-INE être-PRÉT-3 Liisa très souci-PL-INE-POS.3 être-PPR-GÉN ayant l'air «Pendant le déjeuner (En prenant [son] repas de midi), Liisa avait l'air très inquiet. »

(J. Aho, 1884[2011], Rautatie, p. 64)

12b)

hän sö-i puolis-ta

elle manger-PRÉT-3 repas de midi-PAR

« elle prenait [son] repas de midi, elle déjeunait »

12c)

puolise-n syö-minen

repas de midi-GÉN manger-NMLS.NOM

« [la] prise du repas de midi, [le] fait de déjeuner »

De par leur antéposition à l'infinitif, ces compléments se comportent comme les déterminants du nom (l'ordre des éléments étant déterminant-déterminé dans un groupe 
nominal) ; d'un autre côté, leur forme est identique à celle qu'ils ont auprès d'un verbe fini (cf. les ex. 11b et 12b). On note en particulier la forme du complément d'objet qui peut être au cas partitif pour indiquer une valeur quantitative et aspectuelle, à savoir l'incomplétude du procès. Cette possibilité de se construire avec un objet au partitif distingue l'infinitif en TESSA des noms déverbatifs avec lesquels le complément d'objet de la base verbale subit le même type de transposition que la forme sujet (ex. 12c).

Pour récapituler, dans les variétés non standard du finnois, l'infinitif en TESSA forme une construction légère qui comprend rarement plus d'un complément et où l'absence de marquage explicite de l'argument sujet est un trait fréquemment attesté (Ikola, Palomäki et Koitto, 1989, p. 331 ; Lindén, 1961, p. 195).

\subsection{Construction phrastique dans le finnois écrit standard}

Le degré de phrasticité élevé qui caractérise la construction temporelle en TESSA dans le finnois écrit standard est résultat d'une évolution que cette forme a connue surtout au XIX siècle, mais dont les premiers jalons remontent au $\mathrm{XVI}^{\mathrm{e}}$ siècle. Les phénomènes ayant contribué à cette évolution sont plusieurs : contacts de langues dans le contexte de la traduction de textes, notamment de textes religieux, élaboration d'une norme prescriptive du finnois et diversification des genres de textes écrits (cf. Lindén, 1966 ; Sauvageot, 1973, p. 173-174; Itkonen-Kaila, 1991; Herlin et Kotilainen 2005, p. 270-284).

Le changement a concerné tout d'abord les contextes d'emploi de l'infinitif. Dans le finnois écrit, la forme en TESSA peut faire partie de l'apport de l'énoncé (Lindén, 1971). Deuxièmement, cette modification de statut informationnel se voit dans la réalisation des compléments et des modifieurs de la base verbale, qui peuvent être plusieurs et de différents types, et qui sont alors postposés à l'élément recteur. Dans l'exemple (13), l'infinitif katsoessaan «en regardant» est accompagné non seulement d'un complément d'objet postposé, (...) Hollywood-elokuvia ja Disney-piirrettyjä «des films hollywoodiens et des dessins animés de Disney (...) », mais aussi d'un complément au cas essif, pikkutyttö-nä « [quand elle était] petite fille », qui donne des informations sur l'état du sujet de l'infinitif au moment où il est impliqué dans le procès <regarder> et qui s'interprète avec une valeur temporelle :

\section{3)}

Toivoniemi on rotukissaharrastaja,

« Toivoniemi est une amatrice de chats de race»

joka ihastu-i siamilaiskisso-i-hin katso-e-ssa-an pikkutyttö-nä

qui tomber amoureuse-PRÉT-3 -siamois-chat -PL-ILL regarder-INF-INE-POS.3 petite-fille-ESS

« qui est tombée amoureuse des chats siamois quand elle était petite fille, en regardant »

1950-luvu-n Hollywood-elokuv-i-a ja Disney-piirretty-j-ä.

1950-années-GÉN Hollywood-film-PL-PAR et Disney-dessin animé-PL-PAR

« des films hollywoodiens et des dessins animés de Disney des années 1950. »

Ensimmäisen kissansa hän sai 12-vuotiaana ja (...)

«Elle a eu son premier chat à l'âge de 12 ans et (...)»

(Helsingin Sanomat, 9/3/2013)

Le développement vers une construction phrastique a concerné aussi la réalisation de l'argument sujet qui est devenue plus systématique qu'elle ne l'est dans les registres non standard (Kiuru, 1981, Herlin et Kotilainen, 2005, p. 264 ; Ikola, Palomäki et Koitto, 1989, p. 331). En finnois standard, l'absence de marquage de l'argument sujet se limite à des cas où il s'agit d'une interprétation arbitraire, générique (voir ISK, 2004, § 547; cf. l'ex. 5a) : 
14)

Kroppa rentoutu-u tanssi-e-ssa.

corps se détendre-3 danser-INF-INE

« Le corps se détend quand on danse. » (ISK, 2004, § 547)

Quant à un argument sujet à référence non générique, mais lexicalement non spécifié, la langue standard a généralisé l'emploi d'une marque verbale dite passive, $T A$, qui précède la marque de l'infinitif (ex. 15a); la même marque s'utilise avec un verbe fini (ex. 15b) :

15a)

1 Astu-tta-e-ssa ulos asema-huone-en avara-sta ove-sta, aller-PAS-INF-INE dehors gare-hall-GÉN grande-ÉLA porte-ÉLA

«Lorsqu'on sortit du hall de la gare par la grande porte,

2 näytti Helsinki ottavan tulijoita vastaan kuin kunniavieraita.

« on eut l'impression que Helsinki accueillait les arrivants comme des invités d'honneur. »

(J. Aho, 1889, Helsinkiin)

15b)

Kun astu-tt-iin ulos...

lorsque aller-PAS-PRÉT dehors

« Lorsqu'on sortit... »

La réinterprétation verbale de la forme en TESSA n'a pourtant pas changé la réalisation d'un argument sujet spécifique qui se fait à l'aide d'une construction génitivale ou d'un suffixe possessif (voir l'ex. 13 ci-dessus). Ainsi, la construction temporelle constitue une forme hybride combinant des propriétés verbales et nominales (Creissels, 2006, p. 228).

\subsection{Le déterminant démonstratif siinä}

Il existe encore une façon de doter l'infinitif d'une valeur référentielle spécifique, que l'on observe dans des données non standard. La forme en TESSA peut s'employer avec un démonstratif de type siinä qui est au cas inessif et exerce une fonction déterminative :

16)

$\operatorname{siinä} \ddot{a}^{7}$ (DÉM.INE) + base verbale en TESSA (V-INF-INE)

Nos observations sur ce type de syntagme s'appuient sur un corpus de 260 occurrences relevées dans des textes littéraires, souvent marqués par des traits d'oralité, mais aussi sur des forums de discussion sur internet, dans l'édition numérique du quotidien Helsingin Sanomat ainsi que dans les archives de corpus dialectaux. Les textes littéraires que nous avons dépouillés datent pour la plupart de la fin du XIX et du début du $\mathrm{XX}^{\mathrm{e}}$ siècle, mais comportent aussi un roman contemporain ${ }^{8}$.

\footnotetext{
${ }^{7}$ À noter que siinä, inessif du démonstratif se, est issu d'une formation irrégulière : on y observe, au lieu du suffixe $s s A(<s+n A)$, un allongement de la voyelle finale de la base si- et l'élément nä (cf. ISK 2004, § 101). ${ }^{8}$ Le choix de travailler sur des œuvres littéraires de la fin du XIX ${ }^{\mathrm{e}}$ et du début du $\mathrm{XX}^{\mathrm{e}}$ siècle, période où la standardisation du finnois était en cours, peut être justifié par l'accessibilité de ces textes sous format électronique permettant la recherche automatique de formes, mais aussi par l'intérêt qu'ils présentent dans l'étude d'une structure supposée ancienne, mais toujours utilisée, qui a été ignorée dans la tradition de la description grammaticale du finnois.
} 
Avant d'aller plus loin, quelques précisions s'imposent quant à l'analyse de siinä. En effet, cet élément est susceptible de présenter une ambiguïté syntaxique étant donné que les démonstratifs finnois s'emploient aussi bien comme des pronoms autonomes que comme des déterminants. Il faut alors tenir compte de la possibilité que l'élément siinä antéposé à l'infinitif soit un complément valenciel de la base verbale (cf. la partie 3.1). Cette analyse s'envisage par exemple en (17a) où l'infinitif est un verbe de posture statique, seisoa «être debout (sans bouger) », permettant d'exprimer l'idée de « se trouver quelque part ». On peut alors interpréter siinä comme instanciant un complément locatif statique et se rapportant au référent nommé dans le contexte précédent par le groupe nominal yliopiston nurkalle «à l'angle du bâtiment universitaire » (siinä seisoessa «me trouver sur ce lieu ») :

17a)

Kun tulin yliopiston nurkalle, avautui eteeni tuo joka taholta suurien rakennusten sulkema senaatintori.

«Lorsque je suis arrivé à l'angle du bâtiment universitaire, devant moi s'est ouverte la place du Sénat entourée de chaque côté de grands bâtiments. »

$\begin{array}{lllllll}\text { Siinä } & \text { seiso-e-ssa } & \text { ol-isi } & \text { tietysti } & \text { pitä-nyt } & \text { tuntu-a } & \text { juhlallise-lta. } \\ \text { y-INE } & \text { être debout-INF-INE } & \text { AUX-COND.3 } & \text { sans doute } & \text { devoir-PPA } & \text { sembler-INF } & \text { solennel-ABL }\end{array}$

« [Me] trouver sur ce lieu aurait sans doute dû [me] sembler solennel. »

(J. Aho, 1911, Sanomalehtimiesajoiltani)

D'un autre côté, il est également possible d'interpréter siinä comme ayant une valeur autre que spatiale ${ }^{9}$, plus précisément comme un élément qui contribue à opérer un repérage par rapport à une situation (voir NS, s.v. siinä, II 2) identifiable dans le contexte et que la base verbale de l'infinitif décrit lexicalement (siinä seisoessa «ce moment [où je me trouvais à l'angle du bâtiment universitaire] » en 17b) :

17b)

(...)

Siinä seiso-e-ssa ol-isi tietysti pitä-nyt tuntu-a juhlallise-lta. DEM.INE être debout-INF-INE AUX-COND.3 sans doute devoir-PPA sembler-INF solennel-ABL

« Ce moment [où je me trouvais à l'angle du bâtiment universitaire] aurait sans doute dû [me] sembler solennel. »

Ce type d'ambiguïté ne concerne pas les infinitifs dont la base verbale n'accepte pas de complément à valeur statique de type siinä, comme lähteä «partir » ${ }^{10}$ (voir les ex. 2 et 21 ) (Pajunen, 2001, p. 207-208). Quant à des bases verbales telles que ajatella «penser,

\footnotetext{
${ }^{9}$ Un obstacle apparent à l'analyse de siinä comme déterminant de la forme V-TESSA réside justement dans le fait que cet élément soit habituellement décrit comme ayant une valeur spatiale. La Grande grammaire du finnois (ISK, 2004, § 1061) considère que dans l'exemple suivant :

Miet-i-n sïn̈̈ peilate-ssa-ni (...)

se demander-PRET-1 DÉM.INE se regarder dans le miroir-INF-INE-POS.1

«En me regardant dans le miroir, je me suis demandé (...)»

le démonstratif siinä fonctionne comme un constituant à part entière, juxtaposé à la construction infinitive et désignant le lieu où se trouve le sujet qui se pose des questions tout en se regardant dans le miroir. La position que nous défendons ici est que la valeur spatiale n'est qu'une valeur parmi d'autres que peuvent avoir des formes inessives (voir la partie 2).

${ }^{10}$ lähteä «partir » se construit avec un complément à valeur séparative (jo-sta-kin «de quelque part ») ou directionnelle (jo-nne-kin « pour quelque part»).
} 
réfléchir », katsoa « regarder », rupatella «bavarder », etc., s'il n'est pas exclu en théorie de considérer siinä comme un circonstanciel périphérique, hors valence, cette analyse n'est pas très satisfaisante compte tenu de la fonction textuelle de l'infinitif dans le finnois non standard, qui est d'évoquer une situation connue dans le contexte (cf. Lindén 1961, 203-204) : la référence établie par l'infinitif peut être interprétée comme englobant les coordonnées spatiotemporelles de l'événement.

Le statut du démonstratif en tant que déterminant est fondé sur la nominalité de la forme en TESSA. Il est notable que la structure de l'unité composée par siinä et l'infinitif en TESSA soit conforme au principe qui conditionne les syntagmes nominaux en finnois, selon lequel les déterminants épithètes et démonstratifs s'accordent en cas avec l'élément déterminé. En tant que déterminant dépourvu d'autonomie référentielle, siinä est un élément atone ${ }^{11}$, ce que nous avons pu vérifier avec les données orales relevées dans les corpus dialectaux LaX de l'Université de Turku ${ }^{12}$.

Il reste à définir le rôle du déterminant dans l'individuation de la référence de l'infinitif. Le démonstratif se qui devient siinä au cas inessif (voir la note 7) est l'un des trois démonstratifs du finnois. Il a ceci de particulier que son origo déictique n'est pas le locuteur (Larjavaara, 1990, p. 95-96; Laury, 1997, p. 59). Il exprime que l'identifiabilité du référent est préconstruite dans l'espace intersubjectif : le référent est désigné dans la continuité d'une autre situation (cf. Kleiber, 1994, p. 82-83). Ainsi, le démonstratif se invite l'interlocuteur à établir le lien entre la référence actuelle et la situation dont le référent tire son identifiabilité (Vilkuna, 1992, p. 133-135; Duvallon, 2004) ${ }^{13}$.

Pour illustrer l'effet de sens que siinä crée lorsqu'il accompagne l'infinitif en TESSA, considérons les exemples (18a) et (18b), l'un sans et l'autre avec le déterminant. Dans les deux cas, le contexte précédent comporte la description d'une scène où le protagoniste se réveille après avoir dormi dans un endroit peu conventionnel :

18a)

1 Shemeikalla on nälkä, ja ruumis on maa-te-ssa kuoleutu-nut.

corps AUX-3 dormir-INF-INE s'engourdir-PPA

«Shemeikka a faim, et [son] corps s'est engourdi pendant le sommeil (/pendant qu'il était étendu). »

2 Hän katselee raukeasti ja kyllästyneesti eteensä (...)

«Il regarde devant lui d'un air las et apathique (...)»

(J. Aho, 1911, Juha, p. 113)

18b)

1 (...) ja si-tä lähtö-ä ryhdy-tt-iin sitten teke-mä-än.

et DÉM-PAR départ-PAR commencer-PAS-PRÉT ensuite faire-INF-ILL

« (...) et puis on commença à préparer le départ. »

2 Jäsene-t ol-i-vat puutu-nee-t sïnä maa-te-ssa

membre-PL AUX-PRÉT-3.PL s'ankyloser-PPA-PL DÉM.INE être couché-INF-INE

«[Ses] membres s'étaient ankylosés pendant [tout ce temps] qu'il était couché [sur la pelouse], »

\footnotetext{
${ }^{11}$ Les démonstratifs atones connaissent aussi des emplois où leur fonction se rapproche de celle d'un marqueur discursif (pour le démonstratif prochain à l'inessif tässä, voir Etelämäki, 2006, p. 168-173). Il serait intéressant de s'interroger sur le lien entre le statut de déterminant nominal et celui de marqueur discursif, mais cette problématique dépasse le cadre du présent article.

${ }_{12}$ Adresse de ces corpus : http://syntaxarchives.suo.utu.fi/Murrekorpus/.

${ }^{13}$ L'emploi de l'infinitif en TESSA avec les deux autres démonstratifs, le démonstratif proximal tässä et le démonstratif distal tuossa, ne sera pas analysé ici. Pour la discussion sur l'existence ou non de la catégorie de l'article défini en finnois non standard, voir ISK 2004, § 1408 ; Duvallon 2004 ainsi que les références qui y sont mentionnées.
} 


\section{3 ja kaiken kaikkiaan oli kauttaaltaan rypistynyt olo, (...)}

« et tout compte fait, [il] se sentait complètement délabré, (...)»

(M. Rimminen, 2004, Pussikaljaromaani, p. 162)

La forme maatessa, comportant la base verbale maa(t)- « être couché, dormir », peut être glosée par «pendant le repos, pendant le sommeil ». Elle rappelle l'évènement précédent qui constitue un repère temporel, mais aussi causal pour le changement d'état exprimé par le verbe principal. En (18a) où la forme maatessa s'emploie seule, l'accent est mis sur le type de procès, et non pas sur le participant qu'il implique. La description de l'état du corps du protagoniste se fait d'un point de vue externe.

Dans l'exemple (18b), le déterminant siinä n'ajoute rien à la description lexicale du procès $^{14}$. En revanche, il contribue à la construction d'une valeur référentielle spécifique par le fait de mobiliser l'espace intersubjectif de la scène énonciative. En indiquant que l'identifiabilité du référent est préconstruite dans l'espace intersubjectif, il réactualise, avec l'aide du contenu lexical de l'infinitif, toute la scène qui a été mise en place dans le contexte précédent, où le protagoniste s'est couché sur la pelouse pour sombrer dans un demi-sommeil, après quoi il s'est réveillé pour prendre part au bavardage avec ses copains. La mobilisation de l'espace intersubjectif a pour effet d'impliquer l'interlocuteur dans la construction de la valeur référentielle : il est invité à adopter un point de vue interne sur l'événement rappelé et à s'identifier, le cas échéant, au rôle de l'argument sujet qui n'est pas lexicalisé dans l'énoncé ${ }^{15}$.

Le déterminant démonstratif siinä peut se combiner avec un complément antéposé à l'infinitif instanciant l'argument sujet ou objet de la base verbale ou un autre complément nucléaire, et avec un suffixe possessif indiquant l'argument sujet. Ce dernier est utilisé lorsque les deux procès concomitants ont un support commun, susceptible de les contrôler (cf. partie 3.1.) :

19)

Hän muisti miten kalpeana äiti makasi vuoteellaan ja taputteli häntä poskelle.

«Il se rappelait comment sa mère, toute pâle, était couchée dans son lit et lui caressait la joue. »

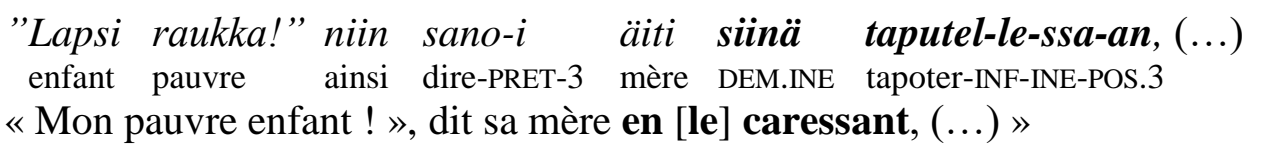

(J. Kokko, 1888, Räisäspoika)

Notons qu'en (19), le verbe taputella « tapoter, caresser » implique aussi un argument objet qui n'est pas instancié dans le groupe nominal siinä taputellessaan « en [le] caressant ». Il peut être récupéré dans la situation qui vient d'être décrite, rappelée par l'infinitif.

\footnotetext{
${ }^{14}$ Le contexte ne motive pas ici l'interprétation de siinä comme un complément locatif de l'infinitif maatessa «être couché ». Notons par ailleurs que l'extrait de texte en $18 \mathrm{~b}$ comporte une autre occurrence du déterminant se à la ligne 1, dans le syntagme nominal qui a pour tête le nom déverbatif lähtö « départ».

${ }^{15}$ Cette dimension intersubjective qui est cruciale dans le fonctionnement déictique du démonstratif $s e$ est sans doute un des facteurs expliquant pourquoi ce type de déterminant est absent des textes informatifs rédigés en langue standard. La caractérisation de la deixis, proposée par Bosredon et Fischer 1992, p. 496, est éclairante ici : « La deixis permet à l'énonciateur et au co-énonciateur de capter un objet en situation selon un processus référentiel qui rajuste perpétuellement cette saisie à partir d'un système de repérage propre à l'un et à l'autre des participants. Cette captation référentielle, rapportée au moment de l'énonciation à la position spatiale et discursive de chacun, crée à chaque prise de parole une perspective nouvelle selon laquelle se construit la référence. Avec la disparition de la prise de parole, ou plus généralement d'un échange impliquant ce type d'interaction, disparaît la nécessité d'une construction référentielle qui procède par positionnement d'une perspective liée aux sujets. » (C'est nous qui soulignons.)
} 
Cependant, le cas de figure le plus intéressant dans notre corpus est celui où le déterminant démonstratif est le seul indice de la valeur référentielle spécifique. L'argument sujet de l'infinitif est alors typiquement non coréférent avec le sujet du verbe principal, comme c'est le cas dans l'exemple (18b). Mais il ne s'agit pas de deux évènements indépendants l'un de l'autre, car le sujet de l'évènement repère est impliqué dans l'évènement repéré d'une manière ou d'une autre, par exemple en tant que siège d'un sentiment ou d'une sensation, telle la sensation d'avoir les membres ankylosés en (18b).

Dans l'exemple (17b) ci-dessus, la prédication principale exprime le sentiment que le narrateur, une fois impliqué dans la situation évoquée par la forme siinä seisoessa «ce moment [où je me trouvais à l'angle du bâtiment universitaire] », était censé éprouver. Le sujet du verbe principal, tuntua « sembler », correspond à ce qui est susceptible de provoquer le sentiment de solennité, mais il n'a pas de matérialisation lexicale. De même, le siège du sentiment reste implicite. Il s'agit d'un participant-expérient non spécifié, nommé personne zéro dans la littérature, dont l'expérience est conceptualisée d'un point de vue à la fois subjective et ouverte au partage, de sorte que l'interlocuteur puisse s'y identifier (Laitinen 1995, 2006).

En plus des prédications sur des sensations et des sentiments, un autre type de proposition principale qui se répète dans notre corpus exprime le fait qu'une idée vienne à l'esprit de quelqu'un (ex. 20) ou disparaisse de sa tête (ex. 21) : Dans les deux exemples ci-dessous, le participant expérient est implicite, dépourvu de matérialisation lexicale dans l'énoncé :

20)

Tyttö kertoi, että reippaasti kävellen matkaan menee puolitoista tuntia, « La fille a dit qu'en marchant bien, il [lui] fallait une heure et demie pour faire le trajet, » ja kaikenlais-ta siinä kävel-le-ssä käy-dä miele-ssä. et toute sorte de chose-PAR avoir le temps-3 DEM.INE marcher-INF-INE passer-INF esprit-INE « et que toutes sortes d'idées avaient le temps de [lui] passer par la tête pendant [s]a marche. »

(Helsingin Sanomat, courrier des lecteurs, 15/1/1998)

21)

1 (A:) - Minkätähden et ole antanutkaan minun ketruuksia ruustinnalle?

«Pourquoi n'as-tu pas donné les fils (que j’avais filés) à la femme du pasteur ?» $(\ldots)$

2 (B:) - Mikä lie tällä kertaa ollut, onhan tuota ennen muistanut... olin minä ruustinnan 3 puheilla,

«Je ne sais pas ce qu'il y a eu cette fois, jusqu'ici j'y ai toujours pensé... j'ai bien parlé avec la femme du pasteur, »

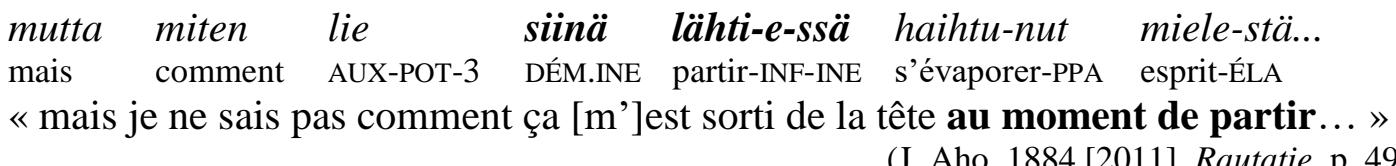

En (21), le deuxième tour de parole (lignes 2-4) est formé de questions que le locuteur B n'adresse pas à son interlocutrice $\mathrm{A}$, mais à un co-énonciateur virtuel qui partage avec lui le point de vue interne sur la scène de départ qui sert de repère pour son oubli.

Enfin, la proposition principale peut exprimer aussi un évènement qui se produit à l'extérieur de l'argument sujet de l'infinitif, tel que l'écoulement du temps. Le sujet non spécifié de l'infinitif est alors un observateur qui doit assumer les conséquences de l'évènement (ex. 22) : 
22)

1 Ilta oli sateinen ja pimeä niinkuin syksyllä ainakin. (...)

«La soirée était pluvieuse et sombre comme souvent en automne. (...)

2 Tuo oli oikein kamalata säätä Hilma-rouvasta, kun hän teki poislähtöä postineidin luota. « Il fait vraiment un sale temps, pensa Madame Hilda qui s'apprêtait à partir de chez la demoiselle de la Poste. »

3 Ja myöhäksikin oli vielä mennyt ilta,

«Et de plus, la soirée était déjà bien avancée. »

4 aika ol-i kulu-nut siinä rupatel-le-ssa niin, temps AUX-PRET.3 s'écouler-PPA DEM.INE baverder-INF-INE de sorte

«Pendant qu'elles bavardaient, le temps avait passé sans »

5 ett-ei si-tä huoman-nut-kaan.

CONJ-NEG.3 le-PAR s'apercevoir-PPA-ADD.NEG

« qu'[elles] s'en aperçoivent. »

6 Kahden kesken olivat istuneet, voudin nuori rouva ja postineiti, jälkimmäisen luona ja

7 jutelleet illan kuluksi tämän maailman asioita.

«Elles s'étaient retrouvées entre elles, la jeune épouse du bailli et la demoiselle de la Poste, chez cette dernière et avaient passé la soirée à causer sur des choses de ce monde. »

(S. Ivalo, 1891, Iltapuhteeksi I)

Aux lignes 2-5 de cet exemple, le narrateur décrit la scène du point de vue du personnage du récit. L'infinitif précédé du démonstratif siinä (ligne 4) exprime un évènement qui est présenté comme préconstruit dans l'espace intersubjectif, mais dont les détails ne sont donnés au lecteur qu'après coup (lignes 6-7).

\section{En guise de conclusion}

Nous avons tenté de montrer qu'en plus de la construction de type phrastique, décrite par les grammaires, l'infinitif en TESSA connaît un emploi nominal, avec un déterminant démonstratif. Lorsque l'individuation référentielle de l'infinitif en TESSA s'appuie sur le déterminant siinä, un rapport étroit se noue entre l'évènement repère et l'évènement repéré malgré l'absence de marquage explicite des participants communs. L'infinitif met en jeu un participant, support du procès, qui a un rôle plus ou moins actif dans l'évènement repère. Ce participant est impliqué aussi dans l'évènement repéré, mais non pas en tant qu'agent qui le contrôle : il s'agit d'un siège du procès ou d'un expérient. La récupération du participant implicite est guidée par siinä qui mobilise l'espace intersubjectif et invite l'interlocuteur à établir le lien vers la première mise en scène de l'évènement repère. Ce point de vue interne sur le procès permet à l'interlocuteur de s'identifier au rôle de l'argument sujet de l'évènement repère et par là même aussi à celui de l'expérient de l'évènement exprimé par le verbe principal.

Ainsi, l'emploi nominal de l'infinitif se distingue de la construction phrastique où les participants du procès sont explicitement mis en scène non seulement par sa forme, mais aussi par le type d'évènement qu'il sert à déterminer et par la façon dont il configure la scène énonciative.

\section{Abréviations}

ABL ablatif ; ADD particule additive ; AUX auxiliaire ; COMP comparatif ; COND conditionnel ; CONJ conjonction ; ELA élatif ; ESS essif ; GEN génitif ; ILL illatif ; INE inessif ; INF infinitif ; INS instructif ; NEG verbe négatif ; NMLS 
marque de nominalisation ; NOM nominatif ; PAR partitif ; PAS passif ; PL pluriel ; POS suffixe possessif ; POT potentiel ; PPA participe passé ; PPR participe présent ; PRET prétérit ; TRA translatif ; 1 première personne ; 3 troisième personne.

\section{Sources des exemples littéraires cités}

Aho, Juhani (1884 [2011]). Rautatie. Kuopio : Icasos.

Aho, Juhani (1911). Sanomalehtimiesajoiltani. Helsinki : Otava.

Aho, Juhani (1911). Juha. Helsinki : Otava.

Aho, Juhani (1889). Helsinkiin. Porvoo : WSOY.

Canth, Minna (1886). Hanna. Helsinki : G. W. Edlund.

Ivalo, Santeri (1891). Iltapuhteeksi I. Porvoo : Werner Söderström.

Rimminen, Mikko (2004). Pussikaljaromaani. Helsinki : Teos.

Kokko, Juhana (1888). Räisäspoika. Porvoo : Werner Söderström.

\section{Bibliographie}

Benveniste, Émile (1966). Problèmes de linguistique générale 1. Gallimard : Paris.

Bosredon, Bernard et Fischer, Sophie (1992). Étiquetage et objets de représentation ou «Ce $\mathrm{N} »$ impossible. In M.-A. Morel \& L. Danon-Boileau (eds.) La deixis. Presses universitaires de France : p. 489-497.

Buridant, Claude (2005). La substantivation de l'infinitif en ancien français : aperçu et perspectives. Langue française, $n^{\circ}$ 147, p. 98-120.

Creissels, Denis (2006). Formes verbales non finies et formes hybrides. In D. Creissels, Syntaxe générale. Une introduction typologique 1. Catégories et constructions. Lavoisier: p. 217-232.

Culioli, Antoine (1999). Pour une linguistique de l'énonciation. Domaine notionnel. Tome 3. Paris : Ophrys.

De Vogüé, Sarah (2006). Qu'est-ce qu'un verbe ? In D. Lebaud, C. Paulin \& K. Poog (eds.) Constructions verbales et production de sens. Presses universitaires de Franche-Comté : p. 43-62.

Duvallon, Outi (2004). Observations sur les déterminants dans les productions orales. In J. Härmä \& U. Tuomarla (eds.) Actes $d u \sigma^{e}$ colloque franco-finlandais de linguistique contrastive. Publications du Département des Langues Romanes de l'Université de Helsinki 15 : p. 75-87.

Duvallon, Outi et de Penanros, Hélène (2015). Schematic form as a theoretical tool for the analysis of prepositions, verbal prefixes and cases in Finnish and in Lithuanian. Uralica Helsingiensia 7, p. 66-94.

Etelämäki, Marja (2006). Toiminta ja tarkoite. Tutkimus suomen pronominista tämä. Helsinki : SKS.

Fernandez-Vest, M. M. Jocelyne (2008). Subordination et degrés de phrasticité dans quelques langues ouraliennes : exemples samiques et fenniques. La linguistique, 44 (2), p. 99-116.

Franckel, Jean-Jacques et Paillard, Denis (2007). Grammaire des prépositions. Tome 1. Paris : Ophrys.

Fuchs, Catherine (1982). La paraphrase entre la langue et le discours. Langue française, $n^{\circ}$ 53, pp. 22-33.

Hakulinen, Lauri (1979). Suomen kielen rakenne ja kehitys. Helsinki : Otava.

Hakulinen, Auli et Karlsson, Fred (1979). Nykysuomen lauseoppia. Helsinki : SKS.

Haspelmath, Martin (1995). The converb as a cross-linguistically valid category. In M.Haspelmath, \& E. König (eds.) Converbs in Cross-Linguistic Perspective. Mouton de Gruyter : p. 1-55. 
Herlin, Ilona et Kotilainen, Lari (2005). Itsenäistyvä infinitiivi: 2. infinitiivin inessiivin kehitys kirjakielen aikana. In I. Herlin, \& L. Visapää (eds.) Elävä kielioppi. Suomen infiniittisten rakenteiden dynamiikkaa. SKS : p. 258-291.

Honeste, Marie Luce (2007). Approche cognitive de la notion de "forme nominalisée du verbe" en français. Faits de langues, $n^{\circ} 30$, p. 59-68.

Ikola, Osmo, Palomäki, Ulla et Koitto, Anna-Kaisa (1989). Suomen murteiden lauseoppia ja tekstioppia. Helsinki : SKS.

ISK = Hakulinen, A., Vilkuna, M., Korhonen, R., Koivisto, V., Heinonen, T. R. et Alho, I. (2004). Iso suomen kielioppi. Helsinki : SKS.

Version électronique : http://scripta.kotus.fi/visk/etusivu.php/.

Itkonen, Terho (1983). Välikatsaus nykysuomen juuriin. Virittäjä, 87, p. 190-229, 349-386.

Itkonen-Kaila, Marja (1991). Agricolan Uuden testamentin temporaalirakenteet: Suomi klassisten ja germaanisten kielten taustaa vasten. Virittäjä, 95, p. 255-280.

Jespersen, Otto (1924). The philosophy of grammar. London: George Allen \& Unwin.

Kiuru, Silva (1981). Temporaalisen lauseenvastikkeen sanajärjestys. Virittäjä, 85, p. 1-20.

Kleiber, Georges (1994). Anaphores et pronoms. Louvain-la-Neuve : Duculot.

Kleiber, Georges (2011). Types de noms: la question des occurrences. Cahiers de Lexicologie, 99, p. 49-70.

Larjavaara, Matti (1990). Suomen deiksis. Helsinki : SKS.

Laury, Ritva (1997). Demonstratives in interaction: the emergence of a definite article in Finnish. Amsterdam : John Benjamins.

Laitinen, Lea (1995). « Nollapersoona », Virittäjäa, 99, p. 337-358.

Laitinen, Lea (2006). Zero person in Finnish : A Grammatical resource for constructing human reference. In M.-L. Helasvuo \& L.Campbell (eds.) Grammar from the Human

Perspective: Case, space and person in Finnish. John Benjamins : p. 209-231.

Lindén, Eeva (1961). Temporaalirakenne suomen murteissa. Virittäjä 65, p. 194-210.

Lindén Eeva 1966. Latinan vaikutus temporaalirakenteeseen suomen kirjakielessä. Virittäjä, 70, p. $1-8$.

Lindén, Eeva (1971). Temporaalirakenne kirjoitetussa kielessä. Virittäjä, 75, p. 23-36.

NS = Nykysuomen sanakirja I-VI. Helsinki : WSOY, 1951-1961.

Pajunen, Anneli (2001). Argumenttirakenne. Asiaintilojen luokitus ja verbien käyttäytyminen suomen kielessä. Helsinki : SKS.

Ravila, Paavo (1945). Nomen verbale suomalais-ugrilaisissa kielissä. Virittäjä, 49, p. 148158.

Saukkonen, Pauli (1965). Itämerensuomalaisten kielten tulosijainfinitiivirakenteiden historiaa I. Johdanto, Adverbiaali infinitiivi. Helsinki : Suomalais-ugrilainen seura.

Sauvageot, Aurélien (1949). Esquisse de la langue finnoise. Paris : Klincksieck.

Sauvageot, Aurélien (1973). L'élaboration de la langue finnoise. Paris : Klincksieck.

Setälä, E. N. (1891). Suomen kielen lauseoppi. Helsinki : K. E. Holm.

Vilkuna, Maria (1992). Referenssi ja määräisyys suomenkielisten tekstien tulkinnassa. Helsinki : SKS.

Vilkuna, Maria (1996). Suomen lauseopin perusteet. Helsinki : Edita. 